\title{
FEATURES OF THE PERIPHERAL IRIS AS SEEN BY GONIOSCOPY AFTER CATARACT EXTRACTION*
}

BY

\author{
J. D. ABRAMS \\ Institute of Ophthalmology and Moorfields Eye Hospital, London
}

Part I. The Anterior Aspect

IT is only to be expected that the main importance of gonioscopy in aphakia is its relevance to glaucoma. Much of the work previously reported has therefore been devoted to the internal appearance and site of the cataract section, and to the presence or absence of peripheral anterior synechiae.

The writer's attention was drawn to the subject from a different direction. During some experimental work on the iris of human cadaver eyes, it was necessary to cut off the corneo-sclera as far into the angle as possible, and an appearance of the iris root was seen which had not been encountered gonioscopically. Fig. 1 shows a human eye in which the entire corneo-sclera has

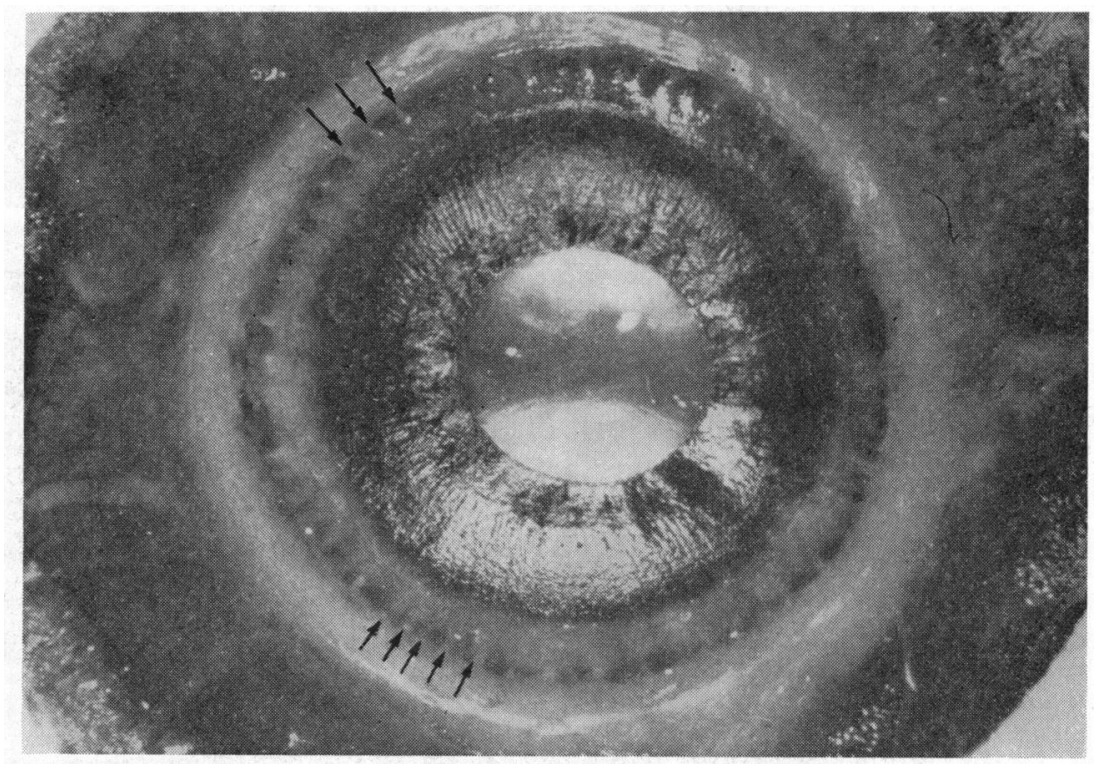

FIG. 1.-Human cadaver eye from which the corneo-sclera has been removed. Arrows indicate the sites of regular thickenings or "teeth" of iris tissue which suspend the iris root from the ciliary body.

been removed. The bulk of the iris is pushed forward by the lens but the extreme periphery shows a regular series of "teeth" by which the iris appears to be attached to the ciliary body. This appearance is so frequent a feature of the dissected eye that the literature was searched for some account of it.

* Received for publication December 19, 1962. 
The region observed is clearly what Fuchs (1885) called the marginal zone of the iris and his own illustrations (Fig. 2) give some hint of a grouping together in bundles of the iris stroma fibres. He describes the bundles as nerve fibres and vessels with crypts in between.

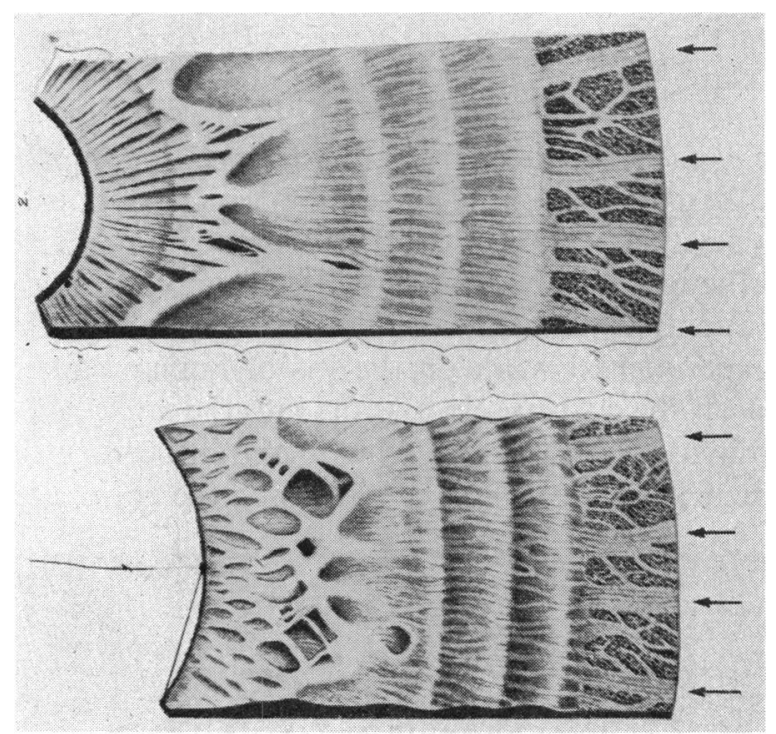

FIG. 2.-Gross anatomy of anterior surface of iris, with pupils of different sizes from Fuchs (1885). Additional arrows indicate the groups of the iris stroma bundles in the marginal zone. Between the groupings are the peripheral crypts. (Reproduced by kind permission of Springer, Berlin.)

It is surprising that the literature of gonioscopy makes scant reference to this appearance. The original work of Salzmann $(1914,1915)$ gives a full anatomical description of the marginal zone, but mentions that it is rarely seen because of the series of elevations which make up the last roll of the iris. Many authors of other classical works on the subject (see Troncoso, 1948; François, 1948; Busacca, 1945) likewise give no illustration of this region of the iris, presumably because it is hidden from view. Trantas (1918) is the only authority who gives a drawing which resembles the anatomical description of the marginal zone (Fig. 3, opposite).

Some years ago a very similar appearance to that in Fig. 1 was seen gonioscopically in a rather unusual case. This was a subject with a total retinal detachment in which, quite spontaneously, the anterior chamber had become extraordinarily deep. The regularity of the processes or teeth recalled to mind the disposition of the ciliary processes but of course one was looking at the anterior surface of the iris.

It was clear that deepening of the anterior chamber should allow easier visualization of the iris root and gonioscopy has been carried out on fifty aphakic eyes. The presence of peripheral anterior synechiae of any extent 


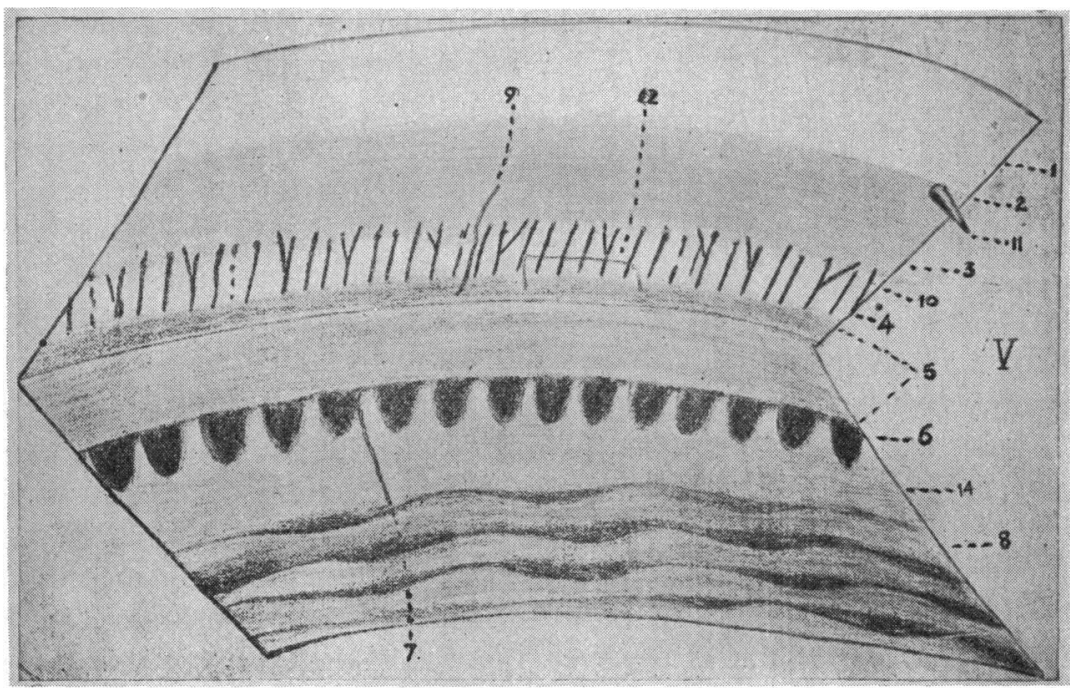

FIG. 3.-Drawing of normal gonioscopic appearance from Trantas (1918). The regularity of the teeth (or of the crypts between them) is indicated at the area marked 6. (Reproduced by kind permission of Masson, Paris.)

will interfere with the visualization of the iris root, but these need not of course be such as to be significant as regards glaucoma. It is usual in aphakia to find a very broad ciliary band, but this was in some cases obliterated by adhesion to it of the peripheral iris with no functional angle closure; that is to say, the canal of Schlemm or the scleral spur was still visible and unobstructed. Even with the presence of some angle closure due to peripheral synechiae it was occasionally possible to see the iris root fully in other parts of the angle. However, eleven cases of the fifty could not be considered as suitable because of the extent of angle closure found.

Of the rest, one third showed, at least in some part of the angle, a picture like that in Fig. 4.

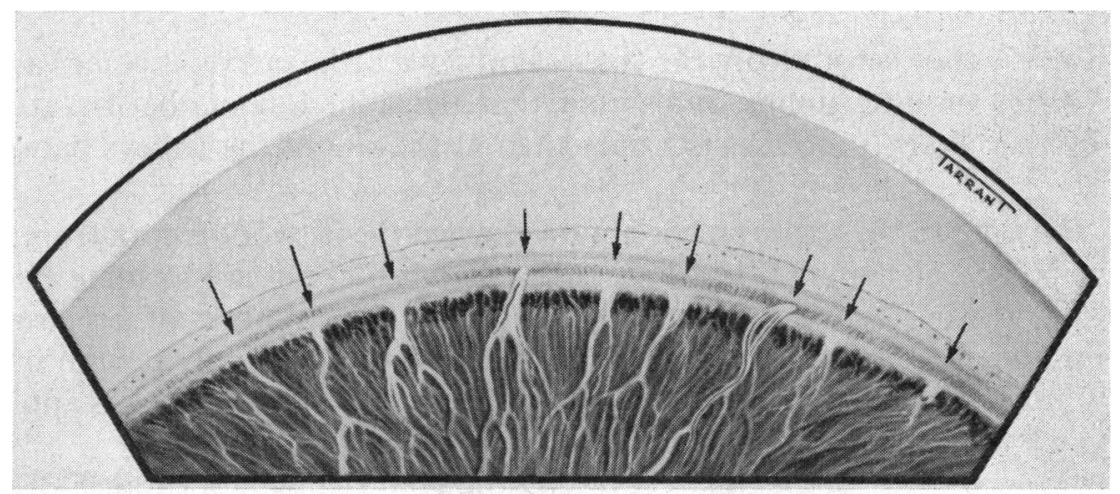

FIG. 4.-Drawing of gonioscopic appearance of an aphakic eye. The spacing of the stroma bundles forming the "teeth", which are arrowed, is a little excessive. They are in fact much closer together. 
The appearance may not be constant throughout the angle and is found more frequently in blue eyes. There is an obvious similarity between the arrangement of the peripheral iris stroma bundles as seen in Figs 1, 2, and 4. Between the bundles, the crypts are visible and it is quite often easy to see what is presumably the posterior pigment epithelium of the iris root in their depths. As is shown, the bundles form a kind of dentate process on a plane anterior to that of the individual stroma fibres lying between the crypts. They cross over to the most posterior part of the ciliary band, which in some cases, as in Fig. 4, is bounded behind by a white band.

The fibre bundles making up a single tooth sometimes contained a bloodvessel, but most often did not. Such a vessel was seen in one or two instances, running directly into the major circle. The individual stroma fibres making up the bundle usually part company from one another after passing into the iris for only a very short distance. Consequently, it is uncommon to find any hint of segmentation of the iris periphery on ordinary slit-lamp examination. Occasionally, however, there is a suggestion of this.

A curious feature of this iris segmentation is that it is more easily seen by rather dim illumination or in immediate proximity to the bright slit beam.

The relationship between these structures and bridging iris processes is uncertain. It may possibly be that this gonioscopic appearance is the result of stretching pre-existent iris processes during surgery. Taken in conjunction with the similar findings in cadaver eyes, however, there may be a relation between the ciliary processes and these teeth.

The inconstancy of the appearance throughout the angle is probably associated with the varying degree of atrophy of the anterior border layer of the iris towards its root, and also, even in the flat iris of the aphakic, with varying development of the last roll of Fuchs.

\section{Part II. The Posterior Aspect}

If the iris root is torn from the ciliary body in a cadaver eye, it comes away in a series of little jumps, indicating that the adhesion of the iris at the site of the ciliary processes is firmer than at the site of the valleys between them.

In referring to the appearances obtained when the iris root is torn from the ciliary body, Salzmann (1912) says: "the ciliary processes are to some extent grown to the back surface of the iris". The bundles of fibres of iris stroma forming the teeth seen in the marginal zone anteriorly certainly remain more firmly attached, probably to the ciliary processes, when light tension is applied to the iris root.

Partial confirmation of this is given by the gonioscopic appearance of the ciliary processes in the iridectomy of some aphakics. It is extremely rare for the iridectomy to be truly basal, as others have shown. In most cases the 
peripheral stump of iris lies somewhat retracted on the base of the ciliary processes, obscuring what relationship there is between them and the iris root. Occasionally, however, a narrow stand can be seen attaching a ciliary process by its base to the back of the iris root. In the case shown in Fig. 5, which is perhaps a little exaggerated, there is a "complete" iridectomy which is not quite basal and the peripheral stump of iris is everted into the incision allowing the iridociliary recess to be turned into view. There are clear connexions between some of the ciliary processes and the iris root.

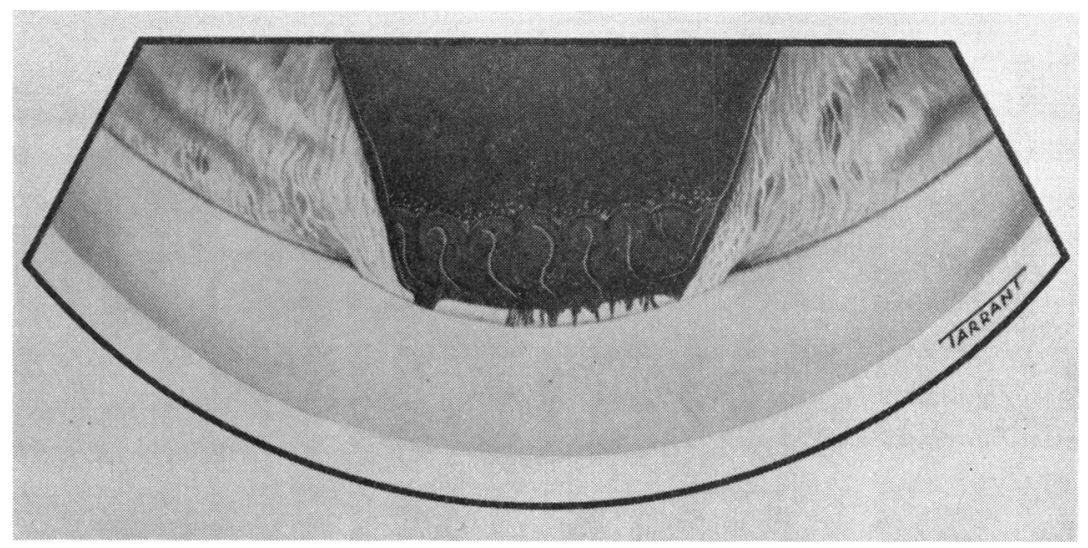

Fig. 5.-See text. Details of neighbouring part of anterior surface of iris not visible because of peripheral synechiae.

It seems reasonable to assume that such connexions would be shown by serial sections of the iris root, and some of these are shown in Fig. 6 (overleaf). These sections are taken from an eye enucleated for a malignant melanoma of the choroid. The anterior segment was normal clinically. Although not shown in the Figures, the sections included a very large pupil $(9 \mathrm{~mm}$.) maximally dilated before operation, and also the optic nerve. It is felt that the presence of a large pupil and of the optic nerve in the section means that any distortion of angle anatomy through obliquity of the plane of cutting is reduced to a minimum. The sections are $6 \mu$ thick and a series of 28 was made up by testing every sixth section, twelve selected sections being shown in Fig. 6 . It is clear by comparing, for example, Sections 3,21 , and 28 , that the relative level of the angles of the posterior and anterior chambers is variable and that the area marked A in Section 21 is typical of a ciliary process "grown to the back surface of the iris".

\section{Discussion}

The visibility or otherwise of the anterior segmented arrangement depends on the plane of the iris being much further back than is usually the case. 

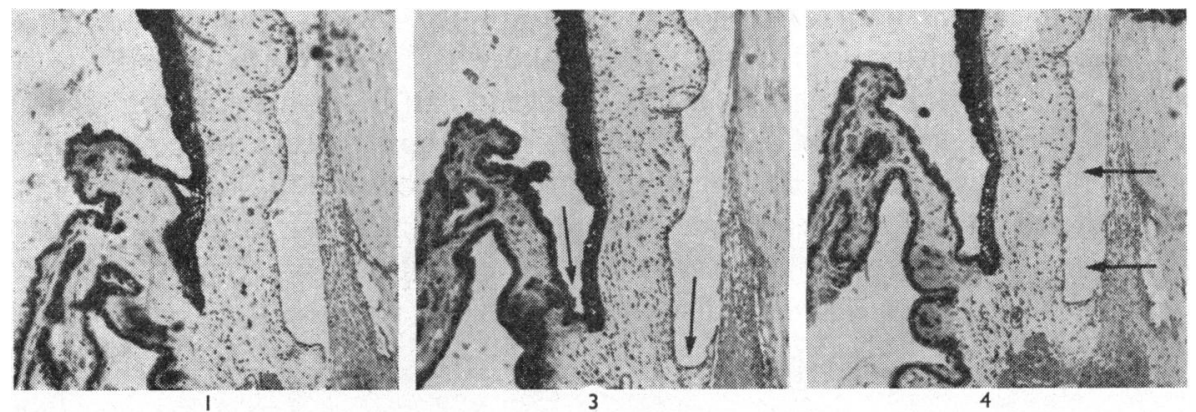

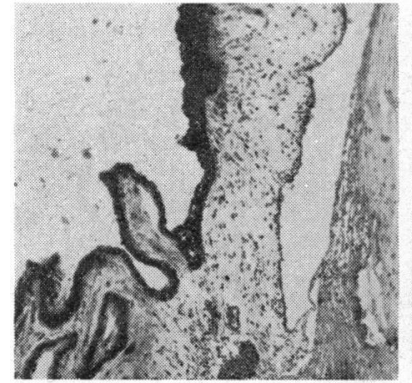

9
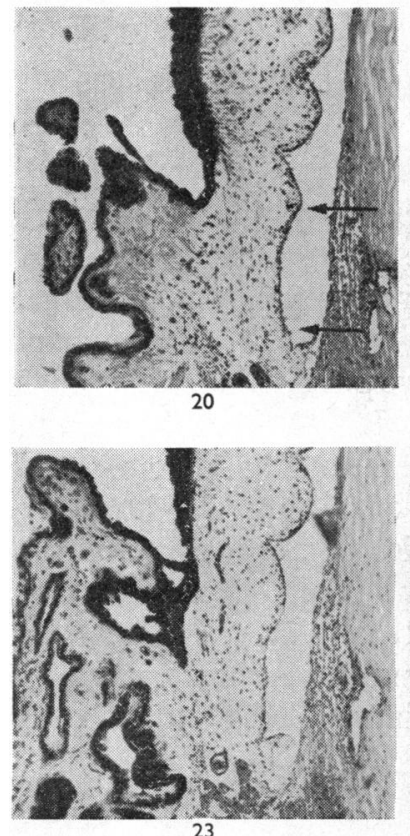

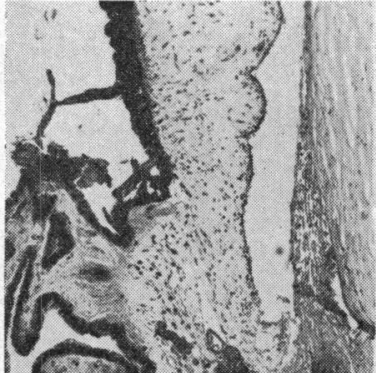

12
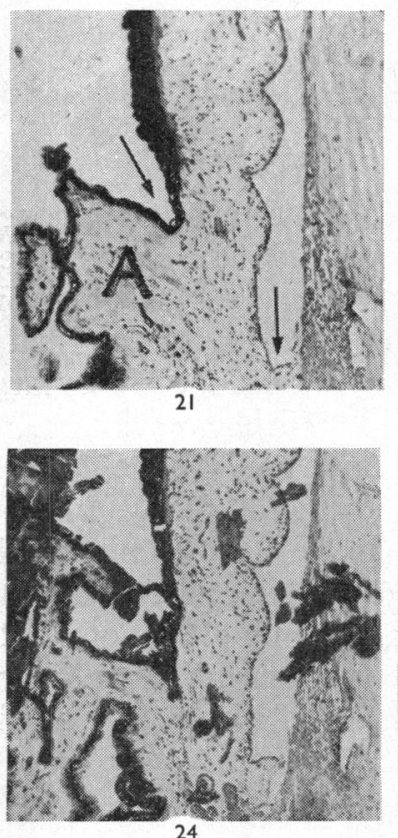

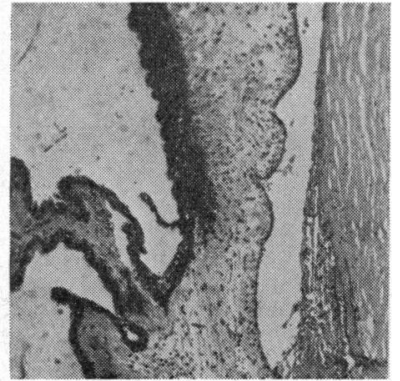

14
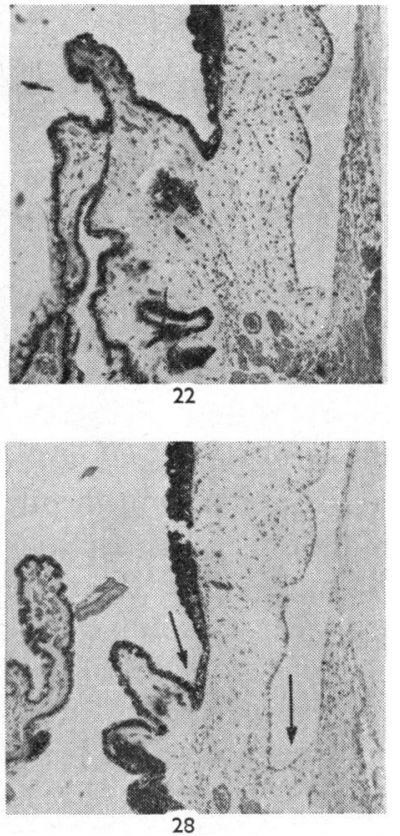

FIG. 6.-Series of sections through irido-ciliary region of an eye with a normal anterior segment (see text). Arrows have been added to Sections 3,21 , and 28 to indicate the varying relation between the angles of the anterior and posterior chambers. The area of the iris between the arrows in Section 4 has become completely filled in by ciliary process in Section 20. (Some of the sections, included for the sake of completeness, are obscured by aberrant lens matter caused by sectioning.) 
The part of the iris considered is behind the last roll of Fuchs, and a simple diagram (Fig. 7) shows why the area is invisible in the phakic eye unless the anterior chamber is very deep indeed.

FIG. 7.-To show the invisibility of the peripheral iris to external or gonioscopic examination in vivo.

Ray 1 indicates that the most peripheral part of the iris seen by external examination is always central to the root because of scleral overhang and the refraction of the cornea.

Ray 2 shows how the last roll of the iris obscures the iris periphery in gonioscopy unless the angle is very wide.

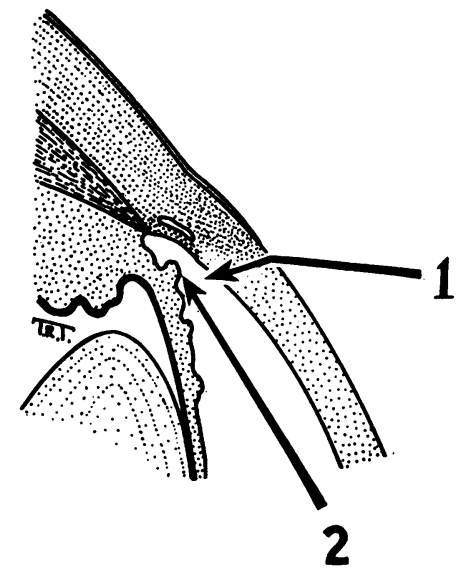

It is significant that only Trantas (1928) has produced an illustration of this appearance gonioscopically and that his method of gonioscopy involved indentation of the limbus, probably pushing the iris root into a more visible position.

The above observations seem to make mandatory a reconsideration of the anatomy of the iris root. While the classical description of the iris root at its thinnest part is undoubtedly true, marked variations are to be expected within regions quite close to one another. The root will obviously be seen in section to be much less thin at the origin of a ciliary process or where one of the anterior stroma bundles exists. The relation between these two is not certain, but if the anatomical description given of the origin of the bloodvessels of the ciliary processes and of the iris from the major arterial circle is valid, then there is at least a basis for suggesting that the anterior stroma bundles which contain the blood-vessels are in certain instances related to ciliary processes.

The segmental arrangement of the peripheral stroma bundles or teeth is sometimes so striking that one is tempted to ask whether the crypts in the iris periphery are really what deserve our attention. It could be that they exist merely by default-default due to the grouping in bundles of the nerves and vessels of the stroma in "preparation" for a segmented attachment of the vessels to the major circle.

In heavily-pigmented irides, a prominent anterior border layer as well as some degree of development of pectinate ligament may obscure the pattern of the iris periphery which is seen in grey or blue eyes.

A further examination of the serial sections in Fig 6, confirms recognized ideas about the shape of the ciliary body in old age; the subject was 73 years old (see Stieve, 1949). The angle appears narrowed because of the maximal 
dilatation of the pupil, but it is clear that the whole of the ciliary body in these sections is a posterior relation of the angle of the anterior chamber. This is not an artefact due, as in many cases, to the appearance of a forward rotation of the ciliary body; there was no cyclodialysis visible in any section of this eye as sometimes appears when the ciliary body shrinks during fixation.

To some extent the view one takes of the events leading to closure of the angle may be influenced by the exact anatomy of the iris root. To consider Section 3, a closed angle would appear to involve apposition of iris to trabeculum. But to consider Section 21, it appears that what must move on to the trabeculum is part of the ciliary body.

\section{SUMMARY}

(1) The gonioscopic visibility of the iris root and the marginal zone, is obscured in most normal eyes by the last roll of Fuchs.

(2) An opportunity to examine this area is afforded by the widened angle of the aphakic eye.

(3) A striking segmentation of the extreme peripheral iris stroma into bundles attached to the ciliary body is seen in some lightly-pigmented aphakic eyes.

(4) The posterior aspect of the irido-ciliary junction is irregular and the bases of the ciliary processes may take tiny origins from the back of the peripheral iris.

(5) The significance of these findings is discussed.

Prof. Norman Ashton kindly supplied the block for the serial sections shown in Fig. 6. The author wishes to thank the many surgeons whose cases were examined. The co-operation is gratefully acknowledged of the Glaucoma Clinic (Dr. J. Gloster), the Department of Anatomy (Dr. C. M. H. Pedler), and the Department of Physiological Optics (Dr. R. A. Weale). Much technical assistance was given by Miss Hazel Jolliffe (histology), Messrs. N. Jeffries and T. Tarrant (illustrations) and Miss Judith Sheppard (typing).

\section{REFERENCES}

BusACCA, A. (1945). "Éléments de gonioscopie normale, pathologique et expérimentale". Tipografia Rossolillo, Saõ Paulo.

FrançoIS. J. (1948). "La gonioscopie". Fonteyn, Louvain.

FuCHS, E. (1885). v. Graefes Arch. Ophthal., 31, abt. 3, p. 39.

SAlZMANN, M. (1912). "The Anatomy and Histology of the Human Eyeball", trans. E. V. L. Brown. University of Chicago Press, Chicago.

(1914). Z. Augenheilk., 31, 1.

- (1915). Ibid., 34, 26.

STTEVE, R. (1949). Anat. Anz., 97, 69.

Troncoso, M. U. (1948). "A' Treatise on Gonioscopy". Davis, Philadelphia.

Trantas, A. (1918). Arch. Ophtal., 36, 257. (1928). Ibid., 45, 617. 